observées ici et sur une tablette en schiste découverte à Alise-Sainte-Reine (Côte-d'Or) ${ }^{47}$. La cupule dans le fond du coffret servait vraisemblablement à malaxer avec un liquide la poudre obtenue par broyage, en fonction du mode d'application du collyre, comme on le verra plus loin.

L'ensemble formé par le coffret à collyres muni d'une cupule, la tablette à broyer et l'étui à instruments permettait d'apprêter les collyres et de les appliquer. Le coffret et la tablette réunis, d'une part, et les instruments à double usage, d'autre part, simplifiaient la trousse de l'oculiste rendue ainsi portative et peu encombrante, surtout si le praticien

47 Musée archéologique de Dijon, inv. 723A. était appelé à se déplacer. Il existait en effet des médecins itinérants, circuitores, selon une tradition remontant aux débuts de la médecine grecque ${ }^{48}$. Hippocrate n'avait-il pas déjà recommandé aux médecins en voyage de se munir d'un matériel facile à transporter?

R. B. et W. M.

48 Les médecins circuitores sont mentionnés dans le Digeste, XXVII, I, 6, 1 ; cf. V. Nutron, Roman Oculists, Epigraphica, 34, 1-2, 1972, p. 16-29. Selon R. Jackson, Doclors and Diseases in the Roman Empire, Londres, 1988, p. 85), la mobilité de certains oculistes correspondait aux besoins des populations rurales; ces oculistes auraient été basés dans des villes.

\title{
LES COLLYRES
}

par Raymond Boyer avec la collaboration de Jean-Noël Barrandon, Corinne Binant, Marie Bui-Thi-Mai, Michel Girard, Bernard Gratuze et Bernard Guineau

Nous avons dénombré 20 collyres à l'état solide répartis dans trois compartiments du coffret d'oculiste; chacun a été affecté d'un numéro. Le quatrième compartiment contenait une petite boucle en fer. Voici la répartition du contenu du coffret (fig. 28) : compartiment A : collyres 1-5; B : collyres $6-15 ; \mathrm{C}$ : collyres $16-20 ; \mathrm{D}$ : boucle 21 .

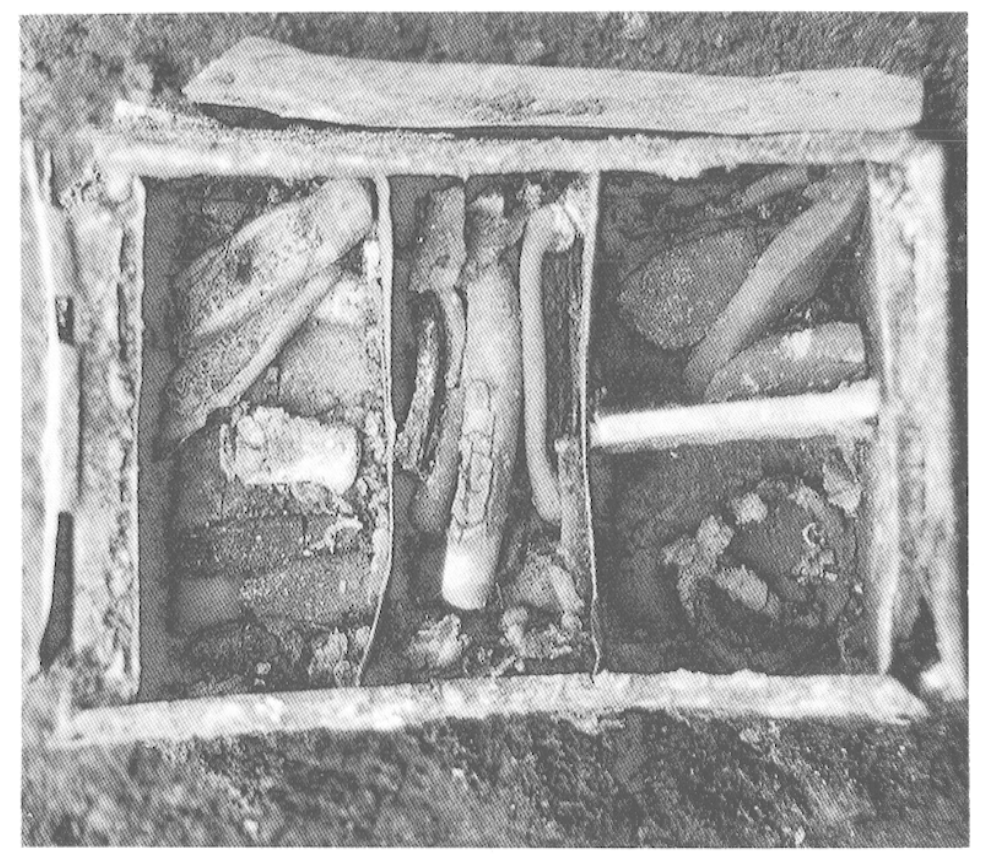

A

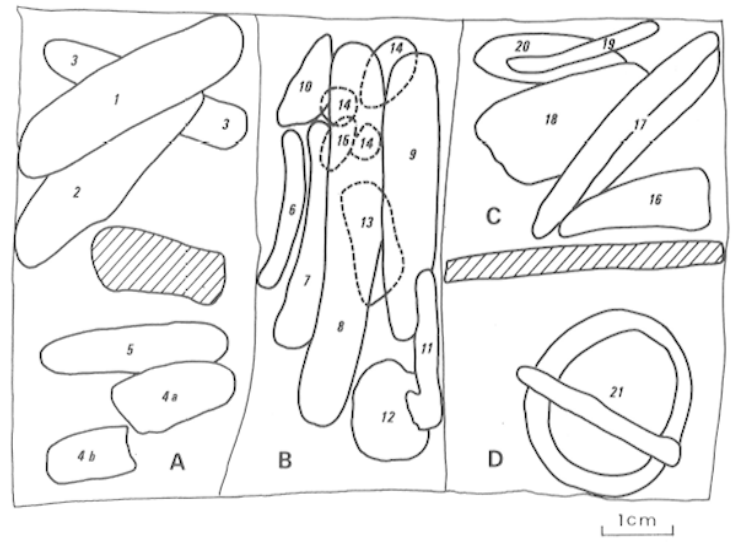

Fig. 28 - A : contenu du coffret lors de la découverte; B : disposition du contenu du coffret : collyres et boucle. 

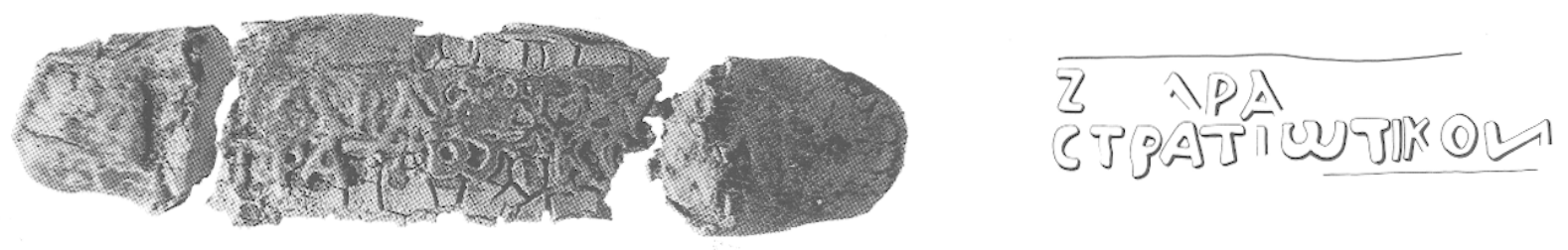

Fig. 29 - Collyre 1.

\section{Remarques liminaires}

La couleur superficielle actuelle des collyres est désignée par référence au Code de E. Séguy et à celui de A. Cailleux ${ }^{49}$.

Les analyses chimiques des collyres, encore inachevées, sont très longues et difficiles. Les résultats provisoires présentés brièvement sont donc particls et incomplets et, dans le cadre limité de cet article, ils sont dépourvus d'argumentation ${ }^{50}$.

\section{COLLYRE 1}

Etat (fig. 29) : intact lors de la découverte; une extrémité brisée lors de l'accident mentionné plus haut. Forme : petit pain oblong. Dimensions : longueur : $42,5 \mathrm{~mm}$; largeur maximale : $10 \mathrm{~mm}$; épaisseur maximale: $3,3 \mathrm{~mm}$. Couleur: surface blanc grisâtre, n' L92 de Cailleux.

Empreinte de cachet : longueur environ : $26 \mathrm{~mm}$; largeur : $6 \mathrm{~mm}$; hauteur des lettres : 2 à $2,3 \mathrm{~mm}$. Texte grec sur deux lignes:

$$
\text { Z[-]ẠPA- - / CTPATIWTIKOИ }
$$

La première ligne se complète ainsi :

$$
\mathrm{Z}[\mathrm{M}] \mathrm{APA}[\mathrm{\Gamma} \Delta \mathrm{OY}]
$$

nom qui se retrouve sur les collyres $2,3,7,9$ et 19 .

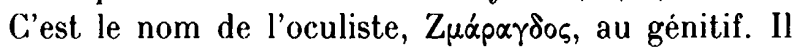
fait partie de la série des anthroponymes tirés des vncables de pierres précieuses, fréquents dans les provinces d'Asie Mineure à l'époque impériale ${ }^{51}$.

49 E. SÉguy, Code universel des couleurs, Paris, 1936 ; - A. Cantr.fux, Code des couleurs des sols, Paris, s.d.

50 Aussi prévoyons-nous la publication d'une étude très complète de ces collyres après achèvement de tous les travaux de laboratoire.

51 L. Ronert, Noms indigènes dans l'Asie Mineure gréco-romaine, Paris, 1963, p. $276 ;-$ cf. aussi W. PAPE, Wörlerbuch des griechischen Eigennamen, $3^{\mathrm{e}}$ éd., Brunswick, 1875, p. 1418; - Frck, Bechtel, Die griechischen Personennamen, 1894, p. 330. Le nom de Smaragdos se rencontre, par exemple, à Milet, à Claudiopolis en Bithynie, à Termessos : Inscriptiones Didyma, $236 \mathrm{C}$ III ; 269, 270; - Dörner, Reise in Bithynien, n. 135; - Tituli Asiae Minoris, III, 1, 56;parmi les gladiateurs : L. RoBert, Gladialeurs dans l'Orient grec, Bibliothèque de l'École pratique des Hautes Études, 278, 1940, p. 301 ; - parmi les éphèbes d'Athènes et dans des inscriptions latines : ibid., p. 276 , n. 7.
La seconde ligne de l'inscription donne le nom du collyre : $\sigma \tau p \alpha \tau \iota \omega \tau \iota x \delta$, connu également sous les formes stratiolides et stratioticum ${ }^{52}$. Cette appellation

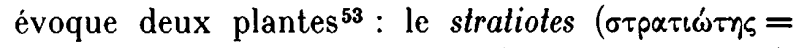
soldat), pistie ou salade du Nil (Pistia stratiotes L.), efficace pour guérir les blessures ${ }^{54}$, et la stratiotice ( $\sigma \tau p \alpha \tau \omega \tau \iota x \eta^{\prime}=$ herbe aux soldats), myriophylle à épis ou volant d'eau (Myriophyllum spicatum L.), possédant les mêmes propriétés que la plante précédente $^{55}$. Cependant, les recettes connues du collyre stratioticum ne comportent pas l'utilisation de ces végétaux.

Analyse chimique : par diffraction $\mathrm{X}$ : cérusite $\mathrm{Pb}\left(\mathrm{CO}_{3}\right)$, composant majeur; hydrozincite $\mathrm{Zn}_{5}\left(\mathrm{CO}_{3}\right)_{2}$ $(\mathrm{OH})_{6}$, des composés à base de cuivre ou de fer $(<5 \%)$ et un composé amorphe comportant une fraction organique.

Analyse pollinique : stérile.

Nous connaissons trois recettes de collyres stratioticum ou stratioticon: une chez Scribonius Largus, deux chez Marcellus ${ }^{56}$. Ces préparations présentent entre elles et avec le collyre 1 des éléments communs, mais aussi des composants différents. Le collyre stratioticon, collyre "militaire" - il existait aussi un mixtum militare ${ }^{57}$ - peut donc désigner divers médicaments en relation avec les facteurs pathogènes auxquels étaient soumis les soldats en particulier : action des intempéries, hygiène insuffisante, méfaits de la poussière des routes ${ }^{58}$;

52 CIL XIII, 10021, 10 et 199.

53 J. André, Les noms de plantes dans la Rome antique, Paris, 1985, p. 250.

54 Pline, N.H., I, 105 et 169 ; - Oribase, Synopsis, 2 , 19.

55 Cf. Dioscoride, IV, 102;-Galien, XII, p. 131 ; - Oribase, XII, s.v., II (éd. Raeder) p. 147.

56 Scribonius Largus, 33 ; - Marcellus, VIII, 123 et 125 .

57 J. Vornor, Inventaire des cachets d'oculistes galloromains, Conférences lyonnaises d'ophtalmologie, 150, 1981, 2, p. $532-533, n^{\circ} 276$.

58 Cf. C. Jullian, Histoire de la Gaule, V, Paris, 1920, p. 29, n. 8 . 

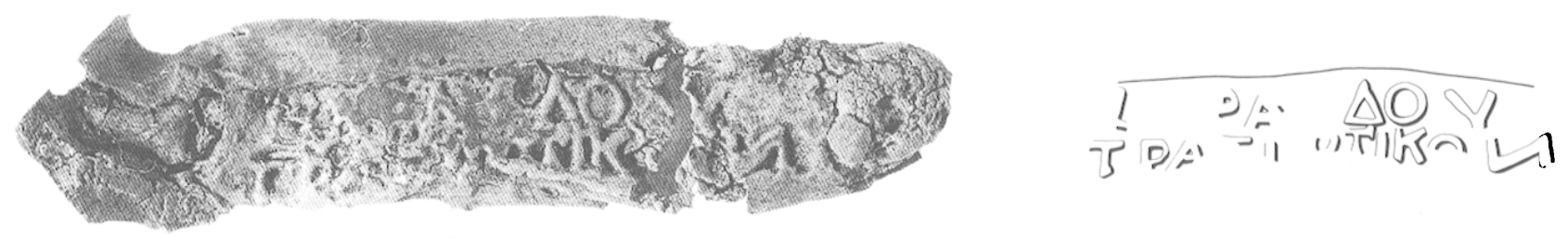

Fig. $30-$ Collyre 2 .

ces derniers sont mentionnés par Marcellus à propos d'un de ses deux collyres stratioticum ${ }^{59}$. Mais il va de soi que l'usage de tels collyres débordait le milieu militaire.

\section{COLLYRE 2}

État (fig. 30) : presque intact lors de la découverte; une extrémité dégradée, surface altérée; brisé en deux parties lors de l'accident. Forme: petit pain oblong. Dimensions : longueur : $46 \mathrm{~mm}$; largeur maximale : $8,6 \mathrm{~mm}$; épaisseur maximale : 4,1 $\mathrm{mm}$; largeur de l'extrémité intacte : う,4 mm. Couleur : surface blanc grisâtre, $\mathrm{n}^{0}$ L92 de Cailleux.

Empreinte de cachel : longueur subsistante : $27,8 \mathrm{~mm}$; largeur maximale : $5,5 \mathrm{~mm}$; largeur à l'extrémité intacte : $4 \mathrm{~mm}$; hauteur des lettres : 2 à 2,4 mm. Texte grec sur deux lignes :

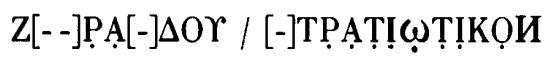

Restitution :

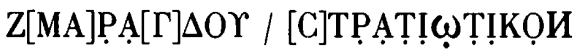

Analyse chimique : non effectuée.

Analyse pollinique : stérile.

L'inscription indique qu'il s'agit du même collyre que le $\mathrm{n}^{\circ} 1$ et du même oculiste.

\section{COLLYRE 3}

Etat (fig. 31) : intact lors de la découverte; brisé lors de l'accident; il subsiste deux fragments très craquelés. Forme: non déterminable. Dimensions du fragment principal : $26 \times 8 \mathrm{~mm}$; épaisseur maximale : $3 \mathrm{~mm}$. Couleur : surface blanc grisâtre, $\mathrm{n}^{\circ}$ L92 de Cailleux.

59 Marcellus, VIII, 123 : Collyrium stratiolicum facil... ad caligines et cicatrices ex itinere et pulvere et fumo collectas; - cf. H. Nifisen, Ancient Ophtalmological Agents, Odense, 1974, p. 16.

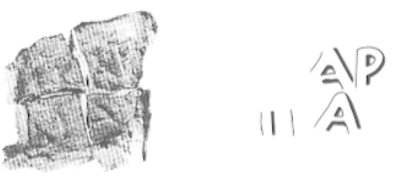

Fig. 31 - Collyre 3 .

Empreinte de cachet : le fragment principal porte une partie d'empreinte; longueur : $11 \mathrm{~mm}$; largeur non mesurable; hauteur des lettres : $2,4 \mathrm{~mm}$. Texte incomplet sur deux lignes :

$$
\text { - - - ]ẠP[- - - / - - ]A[- - - }
$$

L'identité des collyres 1 et 2 trouvés groupés avec le collyre 3 de même couleur, l'identité morphologique et dimensionnelle des lettres et surtout la superposition parfaite (par relevés graphiques et macrophotographiques) des lettres du collyre 3 avec les lettres disposées pareillement sur les collyres 1 et 2 nous autorisent à restituer ainsi l'empreinte :

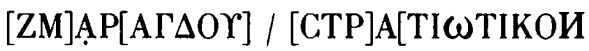

Analyse chimique : par activation neutronique, neutrons thermiques: cuivre $5 \%$, zinc 18,8\%, arsenic $62 \mathrm{ppm}$, antimoine $66 \mathrm{ppm}$, sodium $400 \mathrm{ppm}$, manganèse $550 \mathrm{ppm}$; neutrons rapides : fer $0,4 \%$, plomb $36,2 \%$. Par diffraction $\mathrm{X}$ : cérusite (composant majeur), hydrozincite; brochantite $\mathrm{Cu}_{4} \mathrm{SO}_{4}(\mathrm{OH})_{6}$ dans des grains verts.

Analyse pollinique : stérile.

Ce collyre 3 peut être considéré comme identique aux collyres 1 et 2 .

\section{COLLYRE 4}

État (fig. 32) : fragmenté en deux parties (4a et $4 \mathrm{~b})$; le fragment $4 \mathrm{~b}$ fut perdu lors de l'accident. Forme restituée : petit pain oblong. Dimensions du fragment 4a : longueur : $16 \mathrm{~mm}$; largeur maximale : $8,4 \mathrm{~mm}$; épaisseur maximale : $3,1 \mathrm{~mm}$. Couleur : en surface, brun gris très foncé voisin des $n^{\mathrm{s}} 701$ de Séguy et T771 de Cailleux. 


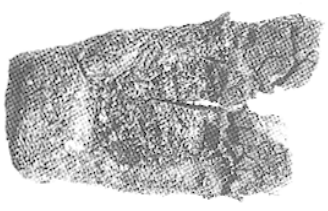

Fig. $32-$ Collyre 4.

Empreinte de cachel : longueur subsistante : $7 \mathrm{~mm}$; largeur maximale : 4,4 mm; largeur à l'extrémité conservée: $3,1 \mathrm{~mm}$; hauteur des lettres : $1,5 \mathrm{~mm}$. Texte incomplet sur deux lignes :

$$
\text { -]M[-- - / AN[-- }
$$

Ce texte était-il grec ou latin?

A la première ligne, une seule lettre peut prendre place avant le M. S'il s'agit ici du nom de l'oculiste, serait-ce encore Z $\mu_{\mu \alpha}^{\prime} \rho \alpha \gamma \delta \circ \varsigma$ ? ou bien seraitce un autre nom?

La seconde ligne semble plutôt comporter le nom du collyre qu'indiquer l'application thérapeutique. Dans ce cas, les collyres connus dont le nom commence par AN... sont : Anacollema, Anethinum, Anicelum, Anodynum, Anthera et Anthemium ${ }^{60}$. Dans l'état actuel de nos recherches, nous ne pouvons proposer une restitution de la seconde ligne de l'empreinte du cachet.

Analyse chimique : par diffraction $\mathrm{X}$ et spectrométrie Raman : zincite $\mathrm{ZnO}$, hydrocérusite (composés majoritaires) et cérusite. Présence possible d'un composé amorphe abondant.

Analyse pollinique : vingt taxons différents sur cinquante pollens déterminés; à noter : le cassis (Ribes nigrum L), des pollens du genre Artemisia et deux pollens d'une Scrofulariacée : l'euphraise (Euphrasia officinalis L.) ${ }^{61}$.

60 Collyres connus par diverses recettes; deux d'entre eux figurent sur des cachets d'oculistes :'anicetum, CIL XIII, $10021,24,65,134,160,195$; - anodynum: ibid., 113; - P. Willeumier, Inscriptions latines des Trois Gaules, $17^{\mathrm{e}}$ suppl. à Gallia, Paris Éd. du CNRS, 1963 (rééd. 1984), p. 214, no 544 ; - J. VoInot, op. cil., p. 488, no $^{\circ} 246$.

61 Nous remercions $M^{\text {mes }} D$. Duzer, N. Planchais et M. J. Maley du Laboratoire de palynologie de Montpellier, ainsi que M. S. Bottema, du Biologisch-Archaeologisch Instituut de Groningen, qui ont bien voulu nous aider à déterminer ce pollen.

\section{COLLYRE 5}

Etat : fragmenté en trois parties; surface très altérée. Forme: petit pain oblong. Dimensions : longueur après assemblage des fragments jointifs : $25,3 \mathrm{~mm}$; largeur maximale : $6,3 \mathrm{~mm}$; épaisseur : 2,4 mm. Couleur: en surface, brun gris voisin des $\mathrm{n}^{0 \mathrm{~s}} 133$ et 134 de Séguy et P51 de Cailleux.

Empreinte de cachet : longueur : $15,5 \mathrm{~mm}$; largeur maximale : $3,6 \mathrm{~mm}$; largeur aux extrémités : 2,9 mm. Lettres complètement effacées.

Analyse chimique : par diffraction $\mathrm{X}$ : zincite, hydrocérusite, et cérusite $\mathrm{Pb}\left(\mathrm{CO}_{3}\right)$, composé minoritaire; présence possible de fer. Par spectrométrie d'absorption infrarouge : spectre très voisin de celui de la gomme adragante.

Analyse pollinique: collyre pauvre en taxons; sur seize pollens, sept appartiennent à l'Euphrasia officinalis L., déjà rencontrée dans le collyre précédent.

\section{COLLYRE 6}

Etat : courbé en arc de cercle, craquelé; une extrémité assez bien conservée. Forme originelle : petit pain oblong. Dimensions : longueur développée : $26 \mathrm{~mm}$; largeur maximale : $6 \mathrm{~mm}$; épaisseur : $2,6 \mathrm{~mm}$. Couleur : brun gris très foncé voisin du $\mathrm{n}^{\circ} \mathrm{T} 51$ de Cailleux.

Empreinte de cachet : sur la face concave du collyre déformé; ses dimensions se sont réduites. Longueur développée : $17 \mathrm{~mm}$; largeur : 1,7 à $3 \mathrm{~mm}$. Texte effacé.

Analyse chimique: par activation neutronique, neutrons thermiques : cuivre $14 \%$, fer $0,5 \%$, plomb $11,2 \%$, étain $0,3 \%$. Par diffraction X : quartz $\mathrm{SiO}_{2}$, calcite $\mathrm{CaCO}_{3}$ et cérusite; présence possible de ténorite $\mathrm{CuO}$ et d'hydrocérusite; un composé organique abondant n'est pas encore identifié.

Analyse pollinique : dominante (soixante-quinze pollens sur quatre-vingt-quatre) des pollens d'Euphrasia officinalis L.; quatre pollens du genre Artemisia.

\section{COLLYRE 7}

Etat (fig. 33) : deux fragments jointifs; une extrémité intacte, l'autre courbée et détériorée; surface altérée. Forme originelle : petit pain oblong. Dimensions : longueur : $37 \mathrm{~mm}$; largeur maximale : $8,2 \mathrm{~mm}$; épaisseur : $3,4 \mathrm{~mm}$. Couleur : en surface, gris voisin du $\mathrm{n}^{\circ} \mathrm{N} 92$ de Cailleux.

Empreinte de cachet : incomplète ; longueur subsistante : $24,5 \mathrm{~mm}$; largeur à l'extrémité intacte : $5 \mathrm{~mm}$; hauteur des lettres : 1,8 $\mathrm{mm}$. Texte grec sur deux lignes: 

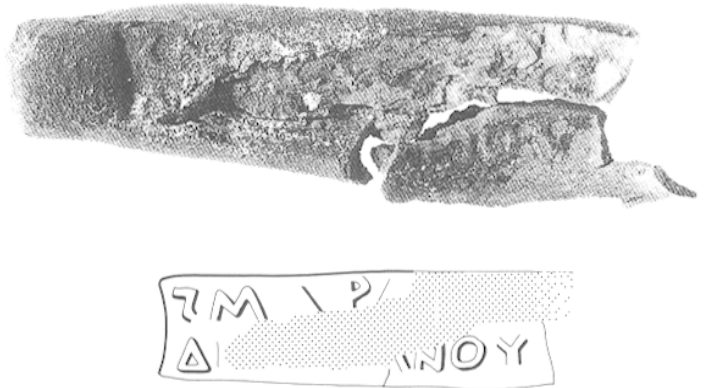

Fig. 33 - Collyre 7.

$$
\text { ZMAPP[- - }] \Delta[--] / \Delta[----] \text { ANOT }
$$

Restitution :

\section{ZMAPP $[\mathrm{A} \Gamma] \Delta[\mathrm{OY}] / \Delta[\mathrm{IA} \Lambda \mathrm{IB}]$ A NOY}

Analyse chimique: par activation neutronique, neutrons thermiques: cuivre $3,8 \%$, zinc $4,5 \%$, arsenic $264 \mathrm{ppm}$, antimoine $530 \mathrm{ppm}$, sodium 900 $\mathrm{ppm}$, manganèse $0,152 \%$, or $6 \mathrm{ppm}$; neutrons rapides : fer $5,8 \%$, plomb $38 \%$. Par diffractométrie $\mathrm{X}$ : hydrocérusite (composé majeur), calcite et quartz (en très faible fraction). En spectrométrie d'absorption infrarouge: présence de gomme arabique et de composés terpéniques; les spectres de ces derniers, comparés à ceux de composés de référence, révèlent la présence de myrrhe.

Analyse pollinique : sur cinquante-six pollens, on en compte cinquante d'Euphrasia officinalis L. et deux du genre Artemisia.

Les collyres $\delta i \dot{\alpha} \lambda_{b} b \dot{\alpha}$ vou ou dialibanum, dont le nom figure sur plusieurs cachets d'oculistes ${ }^{62}$, sont, comme le vocable l'indique, des collyres à base d'encens. Celui-ci est une gommo-oléo-résine contenant notamment des triterpènes ${ }^{63}$ qui lui confèrent des propriétés anti-infectieuses. Des auteurs de l'Antiquité lui reconnaissent plusieurs actions ${ }^{64}$ permettant diverses applications ophtalmiques ${ }^{65}$. Ils donnent en conséquence différentes recettes dont l'encens constitue le composant commun ${ }^{66}$. Dans le

62 CIL XIII, 10021, 19, 47, 95, 106, 113, 118, etc.

63 Cf. H. Nielsen, op. cit., p. 57-58; - R. R. PARIS, H. Moyse, Matière médicale, II, $2^{\mathrm{e}}$ éd., Paris, 1981, p. 307.

64 Selon Dioscoride, I, 68, l'encens a des propriétés mondifiantes, cicatrisantes, astringentes et hémostatiques. Galien l'emploie dans plusieurs collyres : X, p. 1020; XII, p. 715 .

65 Cf. par exemple les indications figurant sur des cachets d'oculistes : CIL XIII, 10021, 19, 47, 95, 113, 118, 138.

66 Celse, VI, 6, 13; - Marcellus, VIII, 196 et 207 ; - Oribase, Synopsis, III, $125 ;$ V, p. 874. collyre 7, la myrrhe a été identifiée. Or, d'une part, il est malaisé de la distinguer de l'encens, d'autre part, ces deux substances ont pu coexister dans un même collyre, comme l'attestent, par exemple, des recettes de Celse ${ }^{6 ?}$.

\section{COLLYRe 8}

État : trouvé presque intact; une extrémité très altérée ; fragmenté lors de l'accident. Forme originelle : bâtonnet cylindrique. Dimensions : longueur restituée d'après cliché pris in situ : environ $46 \mathrm{~mm}$; diamètre d'un fragment : $5,7 \mathrm{~mm}$. Couleur: en surface, vert voisin du $n^{\circ} 403$ de Séguy; des zones brun-rouge sur quelques débris.

Empreinte de cachet : néant.

Analyse chimique : par spectrométrie de fluorescence $\mathrm{X}$ : deux éléments majeurs, cuivre et plomb, et deux mineurs, zinc et fer. Par activation neutronique, neutrons thermiques: cuivre $27,3 \%$, zinc $2,9 \%$, arsenic $400 \mathrm{ppm}$, antimoine $820 \mathrm{ppm}$, sodium $710 \mathrm{ppm}$, manganèse $78 \mathrm{ppm}$, or $26 \mathrm{ppm}$; neutrons rapides : fer $0,6 \%$, plomb $1 \%$. Par diffraction $\mathrm{X}$ : malachite $\mathrm{Cu}_{2}(\mathrm{OH})_{2}\left(\mathrm{CO}_{3}\right)$, cérusite, ténorite et quartz; présence possible d'une argile.

Analyse pollinique : stérile.

\section{COLLYRE 9}

État (fig. 34) : intact lors de la découverte; très fragmenté lors de l'accident. Forme originelle : petit pain oblong. Dimensions : longueur restituée d'après cliché pris in silu : environ $35 \mathrm{~mm}$; largeur maximale d'une extrémité conservée : $9,9 \mathrm{~mm}$; épaisseur : $3 \mathrm{~mm}$. Couleur : en surface, rouge sombre voisin des $\mathrm{n}^{\text {os }} 102$ de Séguy et $\mathrm{S} 15$ de Cailleux.

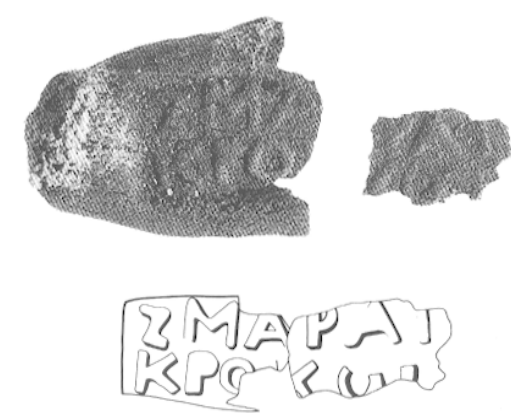

Fig. 34 - Collyre 9 .

67 Celse, VI, 6, 13 : collyre dia libanu contenant un poids égal d'encens et de myrrhe; VI, 6, '25. 
Empreinte de cachet : deux fragments jointifs portent le début d'un texte grec sur deux lignes :

$$
\text { ZMAPAT[---] / KPỌḲ } \omega[---
$$

Longueur : $17 \mathrm{~mm}$; hauteur des lettres : $2 \mathrm{~mm}$. Le traitement en laboratoire d'un cliché pris in situ assure la restitution :

\section{ZMAPAT $[\Delta \mathrm{Or}] /$ KPỌK $\omega[\Delta \mathrm{HC}]$}

Analyse chimique : par activation neutronique, neutrons thermiques: cuivre $3,8 \%$, zinc $3,5 \%$, potassium 2,65\%, arsenic $410 \mathrm{ppm}$, antimoine 90 $\mathrm{ppm}$, sodium $0,157 \%$, manganèse $190 \mathrm{ppm}$, or 1 ppm; neutrons rapides : fer $23 \%$, plomb $0,9 \%$, silicium $4,8 \%$, aluminium $0,75 \%$. Par diffraction $\mathrm{X}$ : hématite $\mathrm{Fe}_{2} \mathrm{O}_{3}$ (composé majeur), jarosite $\mathrm{KFe}_{2}\left(\mathrm{SO}_{4}\right)_{2}(\mathrm{OH})_{6}$ et quartz. Présence d'un composé organique non déterminé. Par microspectrométrie Kaman : pas de spectre caractéristique des caroténoïdes du safran. que.

Analyse pollinique : pas de pollen caractéristi-

L'inscription de l'empreinte du cachet annonce un collyre au safran, à l'instar des cachets portant le nom crocodes $^{68}$. Le safran, obtenu à partir des stigmates des fleurs du Crocus sativus L., entre dans la composition de plusieurs collyres en raison de ses propriétés astringentes et anti-inflammatoires notées par Dioscoride et Galien ${ }^{69}$. Mais dans le collyre étudié, le safran paraît absent. Ce fait n'cst pas surprenant, car le vocable xpoxẃsins peut désigner un collyre couleur de safran, comme c'est le cas.

\section{COLlyre 10}

Élal : incomplet; deux petits fragments craquelés. Forme: indéterminable. Dimensions des fragments : $7 \times 4 \mathrm{~mm}$ et $8,5 \times 3 \mathrm{~mm}$; épaisseur : $2 \mathrm{~mm}$. Couleur : en surface, brun noirâtre voisin du $\mathrm{n}^{\mathrm{o}} \mathrm{T} 91$ de Cailleux.

\section{Empreinte de cachet : néant.}

Analyse chimique : par activation neutronique, neutrons thermiques : cuivre $5,2 \%$, zinc $2 \%$, arse-

68 CIL XIII, 10021, 14, 28, 32, 66, 80, 86, etc.; J. Vornot, op. cil., p. 460 , no $230 ;$ p. 495 , no $252 ;$ p. 516 , $\mathrm{n}^{\prime \prime} 267$.

69 Collyres: Celse, VI, 6,$5 ; 6,6 ; 6,8 ; 6,9$, etc. Scribonius Largus, $21,23,26,27$, etc.; - Marceldus, VIII, 6-10, 14, etc.; - Oribase, Synopsis, VI, p. 872 et 880 ; Al.fixande de Tralles, Practica Alexandri ÿatros greci, Lyon, 1504, fol. 51. Propriétés du safran : Dioscoride, I, 26 ; - Galien, XII, p. 702. nic $48 \mathrm{ppm}$, sodium $0,129 \%$, manganèse $79 \mathrm{ppm}$, brome $700 \mathrm{ppm}$; neutrons rapides : fer $3 \%$, plomb $2,6 \%$. Par diffraction $\mathrm{X}$ : quartz et un composé argileux (?). Par spectrométrie d'absorption infrarouge: gomme arabique et peut-être des composés terpéniques.

Analyse pollinique : stérile.

\section{COLLYRE 11}

État : contours irréguliers, surface très altérée. Forme originelle : bâtonnet cylindrique (?). Dimensions : longueur : $17,3 \mathrm{~mm}$; diamètre moyen : 2,6 $\mathrm{mm}$. Couleur : en surface, brun-rouge sombre voisin des $n^{\text {os }} 701$ et 702 de Séguy et T50 de Cailleux.

Empreinte de cachet : néant.

Analyse chimique : par diffractométrie X: hématite, quartz, calcitc ct pcut-être aragonite $\mathrm{CaCO}_{3}$.

Analyse pollinique : dominante (douze sur dixsept) des pollens d'Euphrasia officinalis L.

\section{COLLYRE 12}

État : très fragmenté. Forme originelle : l'aspect de certains fragments suggère la forme d'une pastille (trochisque) ${ }^{70}$. Dimensions : indéterminables. Couleur: en surface, brun-gris très foncé voisin des $\mathrm{n}^{\text {os }} 701$ de Séguy et T71 de Cailleux.

\section{Empreinte de cachet : néant.}

Analyse chimique : par activation neutronique, neutrons thermiques: cuivre $8,2 \%$, zinc $4,4 \%$, arsenic $140 \mathrm{ppm}$, antimoine $60 \mathrm{ppm}$, sodium 260 $\mathrm{ppm}$, manganèse $150 \mathrm{ppm}$, or $1,4 \mathrm{ppm}$; neutrons rapides : fer $17,5 \%$, plomb $5,9 \%$. Par spectrométrie de fluorescence $X$ : fer (élément majeur). Par diffraction $\mathrm{X}$ : présence d'un composé amorphe majoritai$\mathrm{re}$, de goethite $\mathrm{FeO} . \mathrm{OH}$, quartz, cérusite, ténorite (?). Par spectrométrie d'absorption infrarouge : gomme arabique, peut-être des composés terpéniques.

Analyse pollinique : dominante (cent sept sur cent trente-huit) des pollens d'Euphrasia officinalis L.; deux pollens de cassis (Ribes nigrum L.) et deux du genre Artemisia.

70 Forme attestee archéologiquement, par exemple S. Bonomi, art. cit., col. 81, no 26 ; - M. Korats, Medicus et chirurgus ocularius de Viminatium, Starinar, 37, 1986, p. 57, pl. II, $2 ;$ p. 58 , pl. III, 3 ; p. 59, pl. IV, no 13 ; - CELSE, V, 17, $\mathfrak{2}$ précise que pastillus et trochiscos sont synonymes. 


\section{COLLYRE 13}

Étal : fragment détérioré, avec extrémité bien conservée. Forme originelle: petit pain oblong. Dimensions : longueur : 17,8 mm; largeur maximale : $8,1 \mathrm{~mm}$; épaisseur : $4,3 \mathrm{~mm}$. Couleur : en surface, brun verdâtre voisin des $n^{\text {os }} 441$ et 442 de Séguy.

Empreinte de cachet : près de l'extrémité subsistante, restes d'une empreinte de $5,5 \mathrm{~mm}$ de large; lettres effacées.

Analyse chimique : par activation neutronique, neutrons thermiques: cuivre $3,8 \%$, zinc $9,2 \%$, arsenic $260 \mathrm{ppm}$, antimoine $95 \mathrm{ppm}$, sodium 265 $\mathrm{ppm}$, manganèse $100 \mathrm{ppm}$; neutrons rapides : fer $8,8 \%$, plomb $36 \%$, silicium $0,4 \%$. Par diffraction $\mathrm{X}$ : cérusite, azurite $\mathrm{Cu}_{3}(\mathrm{OH})_{2}\left(\mathrm{CO}_{3}\right)_{2}$; présence probable d'une argile composée de quartz et de kaolin.

Analyse pollinique : dominante (douze sur dixsept) des pollens d'Euphrasia officinalis L.

\section{COLlyre 14}

Etal : très fragmenté; débris trouvés réunis en trois petits groupes. Forme et dimensions : indéterminables. Couleur : en surface, blanc jaunâtre voisin des $n^{\text {"s }} 199$ et 200 de Séguy et K75 de Cailleux.

Empreinte de cachet : néant.

Analyse chimique : par activation neutronique, neutrons thermiques: cuivre $7,2 \%$, zinc $1,90 \%$, arsenic $20 \mathrm{ppm}$, antimoine $18 \mathrm{ppm}$, sodium $377 \mathrm{ppm}$, manganèse $305 \mathrm{ppm}$; neutrons rapides : fer $15 \%$, plomb $2 \%$. Par diffraction $\mathrm{X}$ : goethite (composé majoritaire), cérusite, hydrozincite, probablement une argile. Par spectrométrie d'absorption infrarouge : gomme arabique et composés terpéniques (peutêtre myrrhe).

Analyse pollinique: stérile.

\section{COLlyre 15}

État : fragmenté. Forme : indéterminable. Dimensions des gros fragments : $9 \times 4$ et $9 \times 3,5 \mathrm{~mm}$; épaisseur : $1,5 \mathrm{~mm}$. Couleur : en surface, brun clair et vert vif; cassures : brun voisin des $n^{\text {os }} 437$ de Séguy, T70 et T71 de Cailleux.

\section{Empreinte de cachet : néant.}

Analyse chimique : par activation neutronique, neutrons thermiques: cuivre $10 \%$, zinc $2,4 \%$, arsenic $26 \mathrm{ppm}$, antimoine $80 \mathrm{ppm}$, sodium $0,1236 \%$, manganèse $80 \mathrm{ppm}$; neutrons rapides : fer $7 \%$, plomb $19,4 \%$. Par diffraction $X$ : cérusite (composé principal), azurite et faible proportion de quartz et de calcite. En spectrométrie Raman et d'absorption visible : un composé non identifié.
Analyse pollinique : sur quatorze pollens, cinq d'Euphrasia officinalis L., un de cassis (Ribes nigrum L.) et cinq d'une graminée.

\section{COLLYRE 16}

État : gros fragment, surface assez lisse; une extrémité en biseau paraît témoigner d'un prélèvement ancien. Forme : bâtonnet cylindrique. Dimensions : longueur : $21,8 \mathrm{~mm}$; diamètre : $8,2 \mathrm{~mm}$. Couleur: en surface, olive pâle voisin du $\mathrm{n}^{\circ} \mathrm{N} 91$ de Cailleux; cassure blanc grisâtre voisin du $\mathrm{n}^{\circ} \mathrm{L} 92$ de Cailleux.

Empreinte de cachet : néant.

Analyse chimique : par diffraction $\mathrm{X}$ : cérusite et hydrocérusite. Par microspectrométrie Raman : calcite et noir de carbone; une raie du spectre non attribuée s'observe pour des composés à base de glucose.

Analyse pollinique : stérile.

\section{COLLyre 17}

État : surface craquelée et fissurée ; une extrémité plane, l'autre en biseau parait témoigner d'un prélèvement ancien. Forme : bâtonnet parallélépipédique. Dimensions : longueur : $34,8 \mathrm{~mm}$; section maximale : 10,2 $\times 5 \mathrm{~mm}$. Couleur : en surface, vert voisin des $\mathrm{n}^{\text {os }} 383$ et 385 de Séguy; zones ocre-rouge foncé dans les fissures.

\section{Empreinte de cachet : néant.}

Analyse chimique : par activation neutronique, neutrons thermiques: cuivre $24,8 \%$, zinc $8,2 \%$, arsenic $1,7 \%$, antimoine $0,12 \%$, nickel $1,1 \%$, sodium $520 \mathrm{ppm}$, manganèse $70 \mathrm{ppm}$, or $6 \mathrm{ppm}$; neutrons rapides : fer $13 \%$, plomb $2 \%$. Par spectrométrie de fluorescence $\mathrm{X}$ : cuivre (élément majeur). Par diffraction $\mathrm{X}$ : zincite $\mathrm{ZnO}$ et cuprite $\mathrm{CuO}_{2}$ (composés les plus abondants), calcite, quartz, réalgar $\mathrm{As}_{2} \mathrm{~S}_{2}$. Par spectrométrie Raman : réalgar et malachite.

Analyse pollinique : pas de pollen caractéristique.

\section{COLLYRE 18}

État (fig. 35): deux fragments jointifs, une extrémité mutilée, l'autre intacte. Forme : petit pain oblong. Dimensions : longueur : 27,2 mm; largeur maximale : $12,8 \mathrm{~mm}$; épaisseur : $2 \mathrm{~mm}$. Couleur : blanc jaunâtre voisin du $\mathrm{n}^{\circ}$ L91 de Cailleux.

Empreinte de cachet : mutilée à gauche; longueur subsistante : $27 \mathrm{~mm}$; largeur : $10 \mathrm{~mm}$; hauteur des lettres : $3 \mathrm{~mm}$. Texte latin sur deux lignes: 

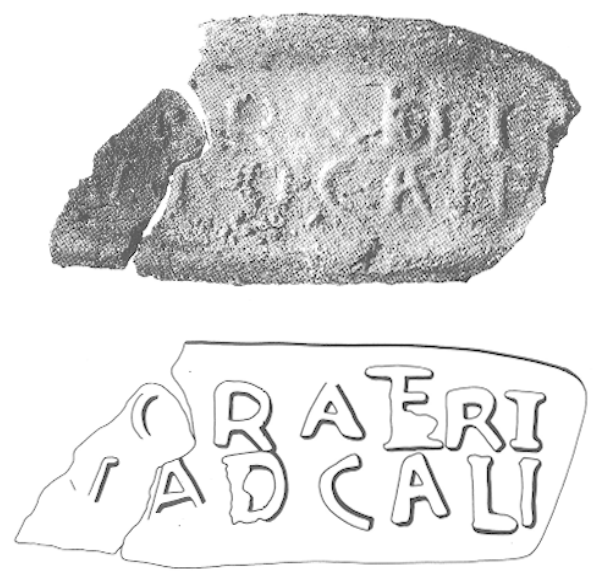

Fig. $35-$ Collyre 18 .

\section{---]ỌR AT్RI / ...-]V AD CALI}

La première ligne comportait sans doute les tria nomina de l'oculiste; on peut restituer seulement:

\section{$[\mathrm{C}] \mathrm{OQR}(\mathrm{NELII})$}

$\mathrm{Au}$ cognomen, ligature de $\mathrm{T}$ et $\mathrm{E}$ et empâtement accidentel à la base de $\mathrm{E}$.

A la seconde ligne subsiste la dernière lettre du nom du collyre : $\underline{\mathrm{Y}}$, probablement $\mathrm{Y}(\mathrm{M})$, suivie de l'indication thérapeutique : AD CALI(GINEM).

Analyse chimique : par activation neutronique, neutrons thermiques: cuivre $5,5 \%$, zinc $9,8 \%$, arsenic 9,3\%, manganèse $480 \mathrm{ppm}$; neutrons rapides : fer $0,5 \%$, plomb $11,2 \%$, aluminium $1,5 \%$. Par diffraction $\mathrm{X}$ : hydrocérusite (composé majeur), réalgar, cérusite, calcite, quartz (identifications confirmées par d'autres techniques). que.

Analyse pollinique: pas de pollen caractéristi-

Plusieurs collyres dont le nom se termine en um sont employés ad caliginem ${ }^{71}$. Cette expression paraît désigner un trouble visuel d'origine sénile ou secondaire à une pathologie inflammatoire ou lésionnelle telle qu'une anomalie de la cornée, une cicatrice, une ophtalmie, une taie, etc. ${ }^{22}$.

71 Collyre ambrosium: CIL XIII, 10021, 149, 166;chelidonium : ibid., 149, 166, 230; - diabsoricum : ibid., 31, 45 ; - diachylum: ibid., 178; - diaopobalsamum : ibid., 86; - mixtum : ibid., 169; - opobalsamum : ibid., 77; - stactum : ibid., 163, 184, 186 et MARCELlus, VIII, $194 ;$ - slactum opobalsamatum : CIL XIII, 48, 103, 191.

72 Celse, VI, 6, 34, distingue la caligo de la cataracte et de la mydriase : VI, 6, 35 et 37 . Si tel cachet d'oculiste associe ad caliginem et ad claritatem (CIL XIII, 10021, 221), il s'agit en fait de prescriptions pour deux syndromes distincts. La thérapeutique ad claritatem semble plutôt concerner une

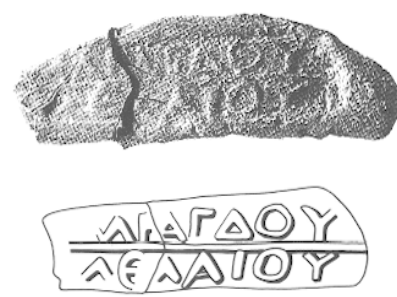

Fig. 36 - Collyre 19.

\section{COLLYRe 19}

Elal (fig. 36) : deux fragments jointifs; une extrémité intacte, l'autre mutilée; surface légèrement altérée. Forme: petit pain oblong. Dimensions: longueur : $19,3 \mathrm{~mm}$; largeur maximale : $5,7 \mathrm{~mm}$; épaisseur : 2,2 mm. Couleur : en surface, noir; cassure, brun voisin du n ${ }^{0}$ P30 de Cailleux.

Empreinte de cachet: incomplète à l'extrémité gauche; longueur subsistante : $16 \mathrm{~mm}$; largeur maximale : $3,8 \mathrm{~mm}$; hauteur des lettres: 1 à $1,3 \mathrm{~mm}$. Texte grec sur deux lignes séparées par un filet mince :

\section{-]MAPAГАOr / -J!AEAAIOT}

Restitution : il n'y a de place que pour une lettre au début de chaque ligne; nous proposons :

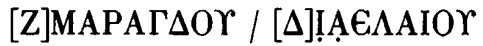

Analyse chimique : par diffraction $\mathrm{X}$ : zincite (composé principal), ténorite, quartz, cérusite (celleci en très faible proportion). Par spectrométrie d'absorption infrarouge : calcite en faible quantité. Par microspectrométrie Raman : gomme arabique et noir de carbone. L'inscription du cachet laisse supposer la présence d'acides gras (voir ci-après); mais les mesures réalisées par chromatographie en phase gazeuse et par spectrométrie de masse donnent des spectres complexes qui appellent de nouvelles recherches.

\section{Analyse pollinique: stérile.}

L'empreinte du cachet indique un collyre à base d'huile (Ẽ $\lambda$ acov) dont le nom grec est à rapprocher de la forme latine dielaeum, sur un cachet d'oculiste ${ }^{73}$.

pathologie de l'accomodation ou de la réfraction à laquelle aurait convenu une correction optique; cf. M.-P. Geste, L'ophtalmologie gallo-romaine d'après l'exemple bourguignon, Thèse de médecine, Dijon, 1981, p. 74.

73 CIL XIII, 10021, 211 : dielaeum len(e) ad siccam lippitudinem ( $=$ ophtalmie sèche). 


\section{COLlyre 20}

État : incurvé, craquelé et très altéré. Forme originelle : bâtonnet parallélépipédique (?). Dimensions : longueur développée : $24 \mathrm{~mm}$; section : $6 \times 2$ à $9 \times 6 \mathrm{~mm}$. Couleur : noir.

Empreinte de cachet : néant.

Analyse chimique : par activation neutronique, neutrons thermiques : cuivre $4 \%$, zinc $1,4 \%$, arsenic $0,78 \%$, antimoine $270 \mathrm{ppm}$, sodium $0,137 \%$, manganèse $150 \mathrm{ppm}$; neutrons rapides : fer $3 \%$, plomb $4 \%$, potassium $0,24 \%$. Par diffraction X : calcite, quartz, une argile probablement du type illite, cérusite, kaolinite et un composé amorphe abondant. Par microspectrométrie Raman : noir de carbone, malachite. Par spectrométrie d'absorption infrarouge : myrrhe.

Analyse pollinique : sur douze pollens, neuf d'Euphrasia officinalis L., un du genre Artemisia et un de l'espèce Xanthium strumarium L. (lampourde antiscrofuleuse).

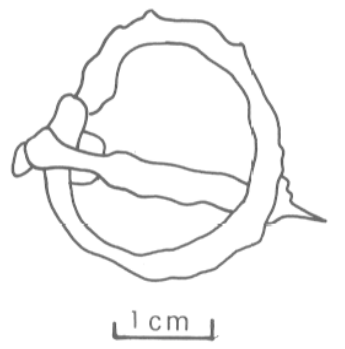

Fig. 37 - Boucle en fer.

\section{OBJET 21}

Le compartiment $\mathrm{D}$ du coffret d'oculiste contenait une boucle circulaire en fer, très corrodée (fig. 37). Diamètre : $24 \mathrm{~mm}$; section : diamètre $3 \mathrm{~mm}$. Un ardillon de $32 \mathrm{~mm}$ de long s'articule sur l'anneau par simple enroulement d'une extrémité. Poids de l'objet : $2 \mathrm{~g}$. Cette boucle n'a pas de lien évident avec l'ophtalmologie.

R. B., B. G., B. G., J.-N. B., C. B., M. B.-T.-M., M. G.

CONCLUSION

par Raymond Boyer et Bernard Guineau

Les collyres contenus dans le coffret d'oculiste se présentent sous trois formes : petits pains oblongs (dix exemplaires), bâtonnets cylindriques ou parallélépipédiques (quatre exemplaires certains), trochisque (un exemplaire probable).

Les collyres en forme de petit pain et un collyre fragmentaire portent tous l'empreinte d'un cachet : huit avec inscription complète ou partielle, trois avec inscription effacée. Sur les huit textes relevés, six sont en grec, un ou peut-être deux en latin. A notre connaissance, c'est la première fois que plusieurs collyres inscrits en grec ont été trouvés groupés ${ }^{74}$.

74 Découvertes isolées de cachets d'oculistes en grec : CIL XIII, 10021, 54, 139, 213, 214, 217. Il y a des cachets d'oculistes dont le texte latin est écrit en caractères grecs, par exemple, ibid., 164; - J.Vornot, op. cit., p. 508, no 261 ; - A. Piganior, Chronique de la $2^{\text {e }}$ circonscription, Gallia, XV, 2, 1957, p. 164.
Sur le plan de l'écriture, des comparaisons très précises entre les empreintes du nom de Z $\mu \alpha \dot{\alpha} \rho \alpha \gamma \delta \circ \varsigma$ révèlent l'identité de celles des collyres 1,2 et 3 ; mais des différences distinguent les empreintes 7, 9 et 19 les unes des autres et du groupe 1-2-3. Il y a

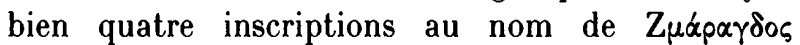
désignant chacune un collyre différent. Ne seraientce pas les empreintes d'un cachet parallélépipédique à quatre faces inscrites, tels que ceux qui sont connus à de nombreux exemplaires?

Sur le plan de la syntaxe, les génitifs d'appartenance $\left(n^{\text {os }} 1-3,7,9,18,19\right)$ désignant les auteurs des recettes de collyres sont abondamment attestés par ailleurs. Les noms des collyres sont exprimés au nominatif ( $\mathrm{n}^{\text {os }} 1-3$ et 9$)$ et au génitif précédé de $\delta i \dot{\alpha}$ $\left(n^{\text {os }} 7\right.$ et 19$)$, selon l'usage fréquent des médecins grecs.

Au point de vue onomastique, le nom de

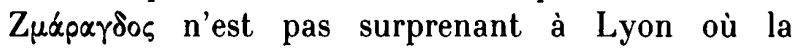
population comptait, dans la seconde moitié du $\mathrm{II}^{\mathrm{e}} \mathrm{s}$. 